Revista de Psicología de la PUCP. Vol. XXI, 2, 2003

\title{
Estudio epidemiológico de los trastornos alimentarios y factores asociados en Lima Metropolitana ${ }^{1}$
}

\author{
Patricia Martínez ${ }^{2}$, Lillyana Zusman ${ }^{3}$, Joan Hartley ${ }^{4}$, Roxanna Morote $^{5}$ \\ y Arturo Calderón ${ }^{6}$ \\ Pontificia Universidad Católica del Perú
}

\begin{abstract}
El presente estudio consistió en analizar la prevalencia de los trastornos alimentarios en la población de mujeres adolescentes escolares de Lima Metropolitana y los factores asociados a dichos trastornos. Asimismo, se buscó determinar la población en riesgo de desarrollar alguno de estos trastornos. Se evaluó un total de 2141 adolescentes entre los 13 y los 19 años, de $3^{\circ}$ a $5^{\circ}$ de secundaria, seleccionadas mediante un muestreo probabilístico bietápico por conglomerados y estratificado según régimen de gestión del colegio (público o privado), y se les aplicó el Inventario de Conductas Alimentarias (ICA) de Zusman (2000). Los resultados mostraron un $16.4 \%$ de casos con uno o más trastornos alimentarios y un $15.1 \%$ de la población en situación de riesgo. Palabras clave: epidemiología, trastornos alimentarios, factores de riesgo, factores asociados, adolescentes.
\end{abstract}

\section{Epidemiologic study of eating disorders and related factors in Lima}

The present study had the objectives of analyzing the prevalence of eating disorders in adolescent women attending school in Metropolitan Lima, identifying the factors related to those disorders and defining the population at risk of developing any of those disorders. A total of 2141 adolescents between 13 and 19 years old in their last three years of high school made up the probabilistic sample. This sample was chosen by clusters in two moments and stratified according to either private or public school. The Eating Behavior Inventory (ICA) was administered. Results showed that $16.4 \%$ of the cases had one or more eating disorders and $15.1 \%$ of the sample was at risk of developing one.

Key words: epidemiology, eating disorders, risk factors, related factors, adolescents.

1 Investigación realizada con el apoyo de la Dirección Académica de Investigación de la Pontificia Universidad Católica del Perú:

2 Magister en Psicología. Profesora Asociada del Departamento de Humanidades de la PUCP. Correo electrónico: pmartin@pucp.edu.pe.

3 Magister en Psicología. Psicoterapeuta. Correo electrónico: maya@interaccess.com.pe.

4 Magister en Psicología Evolutiva Psicoanalítica de Niños y Adolescentes. Profesora de Ciclo Inicial de la PUCP. Correo electrónico: jhartle@pucp.edu.pe.

5 Licenciada en Psicología Clínica. Profesora de la Especialidad de Psicología de la PUCP. Correo electrónico: rmorote@pucp.edu.pe.

6 Estadístico. Profesor de la Especialidad de Matemáticas de la PUCP. Correo electrónico: acalder@pucp.edu.pe. 
Los trastornos alimentarios son entidades clínicas complejas con importantes implicancias físicas, psicológicas y sociales que afectan principalmente a la población femenina. Dichos trastornos se caracterizan por un comportamiento alimentario perturbado así como por una alteración de la percepción de la forma y el peso corporal (APA, 1994). En los últimos 20 años, el conocimiento e investigación sobre los trastornos de alimentación han experimentado un rápido crecimiento y evolución, proporcional al aumento de la incidencia de dichos trastornos así como a su respectiva expansión a todos los niveles socio-económicos. Al respecto, muchos investigadores postulan que éstos han alcanzado proporciones epidémicas.

Actualmente la problemática alimentaria no puede ser comprendida exclusivamente desde un marco individual-intrapsíquico o familiar prescindiendo de variables socioculturales (Malson, 1998; SelviniPalazzoli, 1988; Toro \& Villardel, 1987), en particular, aquellas relacionadas a la estructuración de la identidad femenina (la proporción de mujeres que presenta un trastorno alimentario en relación a los hombres es de 9 a 1). Al respecto, hoy en día diversas sociedades (ubicadas en países con distintos niveles de desarrollo) priorizan valores orientados hacia una figura esbelta y delgada, principalmente en el caso de las mujeres. Striegel-Moore (1993) sostiene que dada la gran presión social existente sobre ellas para que sean atractivas y delgadas uno no debe sorprenderse de que muchas mujeres hagan de la apariencia física y el peso sus más altas prioridades. Las adolescentes del mundo actual están inmersas en una cultura que promueve un modelo de belleza basado en una delgadez poco realista, estableciéndose una escisión cultural donde lo delgado es bonito y lo gordo es feo (Bloom \& Kogel, 1994). Es así como a través de un fenómeno que Fuller (1992) describe como "internalización de la cultura", el cuerpo y la imagen corporal 
Patricia Martínez, Lillyana Zusman, Joan Hartley, Roxanna Morote y Arturo Calderón

son evaluados a la luz de los preceptos culturales actuales que colocan en la delgadez el estereotipo y el "deber ser" de la mujer.

Es interesante observar que el siglo XX (período de cambios profundos en la identidad femenina) se inicia con una patología femenina articulada alrededor del cuerpo, la histeria, y termina con una patología estrechamente vinculada al cuerpo como son los trastornos de alimentación (Zusman, 2000). En esta línea, Gordon (1990) y Malson (1998) afirman que la anorexia nervosa, en particular, refleja la problemática de la mujer de fines del siglo XX, al igual que la histeria en su tiempo reveló la condición de la mujer del siglo XIX.

Diversos autores señalan que el valor excesivo colocado en la delgadez de la mujer, así como la exigencia a cumplir roles contradictorios como el de profesional y madre, aunados al énfasis colocado en la autonomía de la mujer actual, constituyen factores socioculturales importantes en la génesis de los trastornos alimentarios (Bruch, 1973; Coleman, 1985; Gutwill, 1994; Malson, 1998; Perpiñá, 1995; SelviniPalazzoli, 1974; Steiner-Adair, 1986). Sin embargo, es importante notar que la cultura sola no basta para crear un trastorno alimentario ya que ésta se encuentra mediada por las características individuales y el ambiente familiar (Vandereycken \& Meermann, 1984). Los trastornos de alimentación constituyen entidades clínicas cuya causalidad es plurideterminada, donde intervienen múltiples factores predisponentes, precipitantes y de mantenimiento, que entran en juego en un momento determinado.

El DSM-IV (APA, 1994) dentro de su clasificación de trastornos alimentarios incluye la Anorexia y la Bulimia Nervosa así como los Trastornos Alimentarios No Especificados (TANE); en este último grupo se encuentra el Trastorno del Comer Compulsivo o Binge Eating Disorder. El DSM-IV no incluye el trastorno conocido como Yo-yo dieting, en el cual se encuentran aquellas personas que están a dieta intermitentemente, cuyo peso y autoestima suben y bajan, 
Estudio epidemiológico de los trastornos alimentarios y factores asociados...

estando ésta última determinada por el éxito o fracaso de su conducta "dietante" (Zusman, 2000).

Tanto la anorexia como la bulimia nervosa se caracterizan por una excesiva preocupación por los alimentos y por un intenso temor a engordar. Al respecto, algunos autores afirman que dichas entidades comparten el mismo núcleo psicopatológico, pudiendo oscilar o alternarse en una misma persona. (Bloom \& Kogel, 1994; Brusset, 1977; Buena Salud, 1996; Gordon, 1990; Perpiñá, 1995).

La anorexia nervosa se caracteriza por una pérdida de peso autoinducida, disturbios psicológicos y anormalidades fisiológicas secundarias (Schwabe, Lippe, Chang, Pops \& Yager, 1981). Durante muchos años el término anorexia se ha traducido como falta de apetito, sin embargo, Byrne (1987) enfatiza que las pacientes con anorexia nervosa verdaderamente tienen hambre, aclaración de fundamental importancia para el diagnóstico. La persona anoréxica adopta la decisión consciente y rotunda de no comer pues se percibe con sobrepeso (Zusman, 2000). La pérdida de peso se inicia, comúnmente, con una dieta hipocalórica y alta en proteínas (Bruch, 1973; APA, 1994; Toro \& Villardel, 1987).

Duker y Slade (1992) enfatizan la importancia de la actitud anoréxica para el diagnóstico de este desorden y la presencia de negación, por parte de la paciente, de su condición de emaciación (adelgazamiento extremo) y de las consecuencias de la misma, reflejando una delusión corporal que constituye una disrupción del pensamiento. En esta línea, Bruch (1973) resaltó tres síntomas centrales en pacientes anoréxicas: distorsión de proporciones delusionales de la imagen y concepto corporales; distorsión de la percepción o interpretación cognitiva de los estímulos internos, y sentido paralizante de inefectividad. La amenorrea, o ausencia de menstruación, es importante para el diagnóstico diferencial. 
El curso de la anorexia nervosa es altamente variable, aunque éste suele ser crónico. La mortalidad ocurre en el $10 \%$ de los casos y las causas más frecuentes son inanición, suicidio o desbalance electrolítico (APA, 1994; Hsu, 1983; Perpiñá, 1995; Rausch, 1996). Si bien no se puede hablar de un perfil anoréxico universal, se pueden identificar algunas características de personalidad que comúnmente se presentan. La personalidad pre-mórbida de la paciente anoréxica restrictiva es obsesiva, inhibida socialmente, complaciente y cohibida emocionalmente (Wonderlich, 1995), presenta rasgos como perfeccionismo, rigidez, hiperresponsabilidad y sentimientos de ineficacia. Las anoréxicas de tipo atracón/purgante se caracterizan por una mayor impulsividad, reacciones distímicas frecuentes, mayor labilidad emocional y mayor frecuencia de conductas adictivas (Perpiñá, 1995). En general, las pacientes con anorexia nervosa presentan una dificultad para resolver las demandas del proceso de separación-individuación, que son reeditadas en la adolescencia (Rausch, 1996).

La bulimia nervosa se caracteriza por un descontrol real en relación a la comida. La persona que padece este trastorno vive en un ciclo alternante de atracones alimentarios y métodos compensatorios drásticos para evitar la ganancia de peso (APA, 1994; Bloom \& Kogel, 1994; Rausch, 1996).

La detección de este trastorno es una tarea difícil pues la persona intenta ocultar su comportamiento. Así, el problema puede permanecer en secreto por años (Perpiñá, 1995; Rausch, 1996). Esta última autora plantea que el aspecto secreto de la bulimia entraña muchos riesgos. Por medio de las purgaciones, el cuerpo también pierde importantes sustancias para el funcionamiento del corazón, lo que puede ocasionar la muerte. Por lo tanto, algunas veces la bulimia grave es más peligrosa que la anorexia nervosa. Esta enfermedad puede tener un curso crónico o intermitente. 
El atracón alimentario se caracteriza por una pérdida de control. El alimento ingerido durante el atracón suele ser de alto contenido calórico (APA, 1994; Perpiñá, 1995). Los disparadores más frecuentes de un atracón son los estresores interpersonales, los estados de ánimo disfóricos, la presencia o sugestiones de comidas atractivas y engordantes, la privacidad o el aislamiento, y el hambre intenso debido a una restricción alimentaria (APA, 1994; Garfinkel, 1995; Polivy \& Herman, 1993). Por medio del atracón la persona siente alivio, pero éste es temporal. Luego sobrevienen sentimientos de culpa, temor a ganar peso, depresión y sensación de incomodidad física. Si este patrón se vuelve crónico, puede interferir drásticamente con las actividades diarias.

De forma contraria a la anorexia nervosa, el peso de la persona con bulimia no llama la atención, puede ser normal, ligeramente bajo o, incluso, excesivo (APA, 1994; Perpiñá, 1995; Rausch, 1996). Son frecuentes los síntomas de ansiedad, depresión crónica e irritabilidad, pobre control de impulsos, acting-out, promiscuidad, baja tolerancia a la frustración, ideación suicida, conductas de auto-mutilación y abuso de sustancias. Suelen tener una autoestima más baja que el promedio (APA, 1994; Perpiñá, 1995; Polivy \& Herman, 1993; Rausch, 1996; Wonderlich, 1995). Además, presentan trastornos del pensamiento entre los que cabe resaltar el pensamiento dicotómico o del todo o nada pues presentan dificultad para distinguir matices.

Entre un tercio y la mitad de las pacientes con bulimia nervosa cumple el criterio para uno o más trastornos de personalidad, especialmente el Trastorno de Personalidad Limítrofe (APA, 1994). Diversos autores han encontrado que las personas con bulimia y anorexia nervosa, tipo atracón/purgante, tienen un mayor grado de patología y, por lo tanto, peor pronóstico. Del mismo modo, se encuentran mayores niveles de psicopatología en pacientes bulímicas tipo purgante. En muchos casos, la estructura de personalidad de pacientes con bulimia nervosa se asocia a un falso self (Zusman, 2000). 
Las nuevas formas de alimentación socialmente aceptadas y validadas, así como la evidencia clínica con mujeres que padecen trastornos de alimentación, ha llevado a diversos autores a describir lo que puede denominarse como trastornos intermedios (Hartley, 1999; Zusman, 2000). Estos trastornos no cumplen los criterios diagnósticos formales que plantea el DSM-IV (APA,1994) para anorexia y bulimia nervosa pero se asemejan a la alimentación perturbada y al tipo de relación que la persona establece con su cuerpo en estos trastornos. Algunas de estas manifestaciones están incluidas en la descripción que hace el DSM-IV (APA, 1994) de los Trastornos Alimentarios No Especificados (TANE).

Los TANE se pueden dividir en dos grupos: los que se asemejan a la anorexia o bulimia nervosa pero no cumplen todos sus criterios (constituyen los casos subclínicos), y los que presentan una figura clínica particular (Fairburn \& Walsh, 1995). Dentro de los TANE, el DSM-IV (APA, 1994) especifica el Trastorno del Comer Compulsivo (o Binge-Eating Disorder). Este consiste en episodios recurrentes de atracones en la ausencia de comportamientos compensatorios inapropiados característicos de la bulimia nervosa. Una persona que sólo se limita al comer compulsivo sin vomitar, lo más probable es que aumente de peso hasta el punto de la obesidad. Sin embargo, esto dependerá de la frecuencia de dicha conducta (Bruch, 1973, 1994; Rausch, 1996).

Finalmente, aunque el DSM-IV (APA, 1994) no menciona los trastornos yo-yo dieting de forma específica, estos trastornos corresponden a los ejemplos 1 y 2 de los TANE. Los trastornos yo-yo dieting se caracterizan por el proceso de hacer dietas yo-yo, las que alternan períodos de elevada ingesta de alimentos con períodos de abstinencia alimentaria. Lo importante en este grupo no es la cantidad ni la calidad de la comida sino más bien la actitud ante el comer. El yo-yo dieting describe la percepción que tienen las personas de su imagen corporal y autoestima, las cuales oscilan, subiendo y bajando 
Estudio epidemiológico de los trastornos alimentarios y factores asociados...

como el yo-yo, de acuerdo al éxito o fracaso del dieting. Estos trastornos se caracterizan principalmente porque la persona organiza su vida en función de sus dietas y además fracasa repetidamente en su intento de cumplirlas.

Existe un consenso general de que los trastornos alimentarios han aumentado dramáticamente desde 1960. También existe acuerdo entre los investigadores en que la prevalencia de los trastornos alimentarios es mayor en adolescentes y mujeres jóvenes (Malson, 1998). De acuerdo a recientes estudios realizados en Estados Unidos con mujeres entre 15 y 30 años de edad, la prevalencia de anorexia nervosa está entre $0.5 \%$ y $1.0 \%$, la bulimia nervosa entre $1 \%$ y $3 \%$ y los Trastornos Alimentarios No Especificados tienen una prevalencia del $15 \%$ (Abraham \& Llewellyn-Jones, 1997; APA, 1994). Asimismo, Hoek (1995) refiere que los trastornos alimentarios son especialmente comunes en mujeres jóvenes afectando del $2 \%$ al $4 \%$ de la población. Estas cifras adquieren proporciones alarmantes si se considera que entre el $5 \%$ y el $18 \%$ de los casos culminan en la muerte. Cabe mencionar que los casos diagnosticados representan sólo la punta del iceberg de una población más extensa de trastornos subclínicos. En este sentido, Polivy y Herman (1987) sostienen que la dieta y la concurrente mentalidad dietante son descriptivamente y prescriptivamente normativas y, por tanto, la alimentación "normal" de hoy debe ser tratada de la misma forma que una anorexia o una bulimia nervosa.

En nuestro medio, Hartley (1999) encontró que, en una muestra de 361 adolescentes escolares de colegios públicos y privados de Lima, el porcentaje de personas en riesgo de desarrollar un trastorno alimentario estaba entre el $10.5 \%$ y el $15.2 \%$. Zusman (2000) encontró que, en una muestra de 695 adolescentes escolares, también de colegios públicos y privados, el $18.6 \%$ presentaron indicadores de trastornos alimentarios. Finalmente, la Asociación de Lucha Contra la Bulimia y la Anorexia (ALUBA) en un estudio realizado en población universitaria femenina 
de universidades privadas en el 2000, encontró que el $11.3 \%$ de la muestra presentaba anorexia o bulimia y el $43.7 \%$ podría considerarse en riesgo (Agurto, 2000).

A partir de lo descrito hasta aquí, la presente investigación se centró en determinar la prevalencia de los trastornos alimentarios en la población de mujeres adolescentes escolares de Lima Metropolitana. Los objetivos fueron:

- Determinar el Indice de Masa Corporal de la muestra estudiada y ubicar a las participantes en las diferentes categorías de peso (bajo peso severo, bajo peso, peso normal y sobrepeso).

- Determinar la prevalencia de los trastornos alimentarios en Lima Metropolitana de acuerdo a los criterios diagnósticos del ICA.

- Determinar la población en riesgo de presentar trastornos alimentarios en Lima Metropolitana.

- Comparar la incidencia de los trastornos alimentarios de acuerdo al tipo de colegio (público o privado).

- Determinar los factores asociados a la presencia de trastornos alimentarios.

\section{Metodología}

Se trató de un estudio epidemiológico de tipo descriptivo (Bromet, 1989) en el que se buscó determinar la prevalencia de los trastornos alimentarios en la población de mujeres adolescentes escolares de Lima Metropolitana, es decir, el número de personas que presentan el trastorno. El diseño corresponde a lo que Hernández, Fernández y Baptista (1998) definen como un diseño transeccional descriptivo. 
Estudio epidemiológico de los trastornos alimentarios y factores asociados...

\section{Participantes}

En la medida en que se trató de un estudio epidemiológico en el que el procedimiento para la selección de la muestra resultó fundamental para la generalización de los resultados, se describirá en primer lugar la técnica de muestreo utilizada para luego describir a las participantes del estudio.

La población objetivo fue la de alumnas de tercer a quinto año de secundaria de modalidad escolarizada en colegios públicos y privados de Lima Metropolitana. La muestra tomada corresponde a un diseño de muestreo probabilístico bietápico por conglomerados y estratificado según régimen de gestión del colegio (público o privado), con asignación proporcional y selección de alumnos en dos etapas. En la primera etapa de selección se tomó una muestra de colegios dentro de cada estrato y, hecha la selección de colegios, dentro de cada colegio se tomó una muestra aleatoria simple de alumnas distribuidas por grados.

En el momento del diseño de la muestra y usando como marco muestral el Padrón de Centros Educativos del Ministerio de Educación, actualizado a 1999, la población objetivo constaba de 126,710 alumnas, distribuidas en 1,315 centros educativos. El tamaño de muestra mínimo de 2,000 alumnas se calculó de modo que el error de estimación no pasara de $2 \%$ con una confiabilidad de $95 \%$.

Para controlar el efecto de tamaño del centro (número de alumnas), se procedió a estratificar la población de centros educativos (públicos y privados) de acuerdo al tamaño del colegio (hasta 100 alumnas y más de 100 alumnas). Adicionalmente se consideró la ubicación del colegio según distrito, como una aproximación a estratos de pobreza relativa, de acuerdo al mapa de pobreza de FONCODES de 1993, que usa un indicador por distritos tomando valores entre 1.22 y 16 , donde a mayor valor del indicador, mayor pobreza relativa. Para facilitar el diseño, se 
Patricia Martínez, Lillyana Zusman, Joan Hartley, Roxanna Morote y Arturo Calderón

redujo a dos los estratos de pobreza, el primero agrupa los distritos cuyo índice de pobreza está debajo del punto medio del rango posible (8.61) y el segundo agrupa al resto de distritos.

Para el sorteo de colegios, al cruzar los tres criterios anteriormente mencionados, se obtuvo un total de ocho estratos que fueron usados para clasificar los centros educativos y sus alumnas, de acuerdo al Cuadro 1.

\section{Cuadro 1}

Distribución de la población según criterios de estratificación

\begin{tabular}{|l|cc|cc|cc|cc|cc|}
\hline \multirow{2}{*}{$\begin{array}{l}\text { Pobreza } \\
\text { relativa }\end{array}$} & \multicolumn{9}{|c|}{ Público } & \multicolumn{3}{|c|}{ Privado } & \multicolumn{2}{|c|}{ Total } \\
\cline { 2 - 11 } & Hasta 100 & Más de 100 & \multicolumn{2}{|c|}{ Hasta } & 100 & \multicolumn{2}{|c|}{ Más de 100} & & \\
\cline { 2 - 11 } & $f$ & $\%$ & $f$ & $\%$ & $f$ & $\%$ & $f$ & $\%$ & $f$ & $\%$ \\
\hline Baja & 2218 & 1,8 & 54913 & 43,3 & 13991 & 11,0 & 14317 & 11,3 & 85439 & 67,4 \\
Alta & 2247 & 1,8 & 28826 & 22,7 & 6411 & 5,1 & 3787 & 3,0 & 41271 & 32,6 \\
& & & & & & & & & & \\
Total & 4465 & 3,5 & 83739 & 66,1 & 20402 & 16,1 & 18104 & 14,3 & 126710 & 100 \\
\hline
\end{tabular}

Los 2,000 casos iniciales se distribuyeron proporcionalmente entre los ocho estratos y así se obtuvo la cantidad mínima de casos que había que lograr por estrato. Luego, fijando una tasa de 50 alumnas para los colegios pequeños y 100 para los grandes, se redistribuyó la muestra inicial de 2,000 alumnas obteniéndose la cantidad de colegios que había que seleccionar en cada uno de los ocho estratos de sorteo. En previsión de los inevitables rechazos y protocolos anulados, se determinó una mayor cantidad de alumnas en los estratos de colegios pequeños y se reajustó la muestra de modo que el diseño final tenía una estructura aproximadamente proporcional a la estructura de la población (muestra autoponderada), con el fin de facilitar los análisis y evitar el cálculo de ponderaciones adicionales en los mismos. 
Estudio epidemiológico de los trastornos alimentarios y factores asociados...

Finalmente quedó un total de 25 centros educativos a ser muestreados, a razón de 16 centros públicos y 9 privados. De los primeros se tenía que reunir un mínimo de 1,500 alumnas y de los segundos se tenía que lograr un mínimo de 600. También se identificó una "banca" de 13 centros de reemplazo seleccionados aleatoria e independientemente de los 25 colegios de la muestra final. Con ambas listas se procedió al trabajo de campo.

Tomada la muestra y hechos los reajustes por rechazos de algunos centros educativos, se obtuvo la muestra final, cuya estructura figura en el Cuadro 2. Las participantes del estudio fueron en total 2,141 adolescentes entre 13 y 19 años que se encontraban cursando entre el 3ro. y 5to. de secundaria. El promedio de edad fue de 15,3 años.

\section{Cuadro 2}

Muestra final de alumnas y número de colegios según gestión

\begin{tabular}{|lcccc|}
\hline Gestión & Alumnas & $\%$ & Colegios & $\%$ \\
\hline Público & 1555 & 72,6 & 18 & 69,2 \\
Privado & 586 & 27,4 & 8 & 30,8 \\
Total & 2141 & 100 & 26 & 100 \\
\hline
\end{tabular}

El 48,4\% de las participantes (1,037 adolescentes) estudia en colegios segregados, mientras que el restante $51,6 \%$ (1,104 adolescentes) lo hace en colegios mixtos. El $72,63 \%$ de participantes $(1,555$ adolescentes) estudia en colegios de gestión pública mientras que el $27,37 \%$ (586 adolescentes) lo hace en colegios particulares.

En cuanto a las características familiares, se encuentra que 1,460 participantes (el 68,2\%) viven con su familia nuclear completa o con más familiares (madre, padre, hermanos y/o otros), mientras que 680 participantes $(31,8 \%)$ viven con su familia nuclear incompleta (ausencia de madre y/o padre) o sólo con otros familiares. Las adolescentes tienen como peso promedio los $52,19 \mathrm{~kg}$ y como peso ideal promedio los 49,43 
$\mathrm{kg}$. Su estatura real promedio es $1,58 \mathrm{~m}$ mientras que la estatura ideal promedio es $1,67 \mathrm{~m}$.

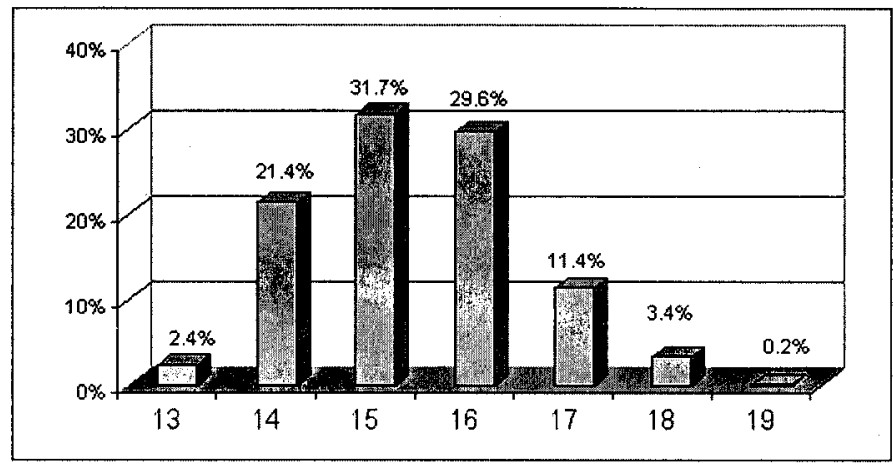

Figura 1. Participantes por edad

\section{Instrumento}

El instrumento utilizado fue el Inventario de Conductas Alimenticias (ICA) de Zusman (2000). Se trata de una escala tipo Likert de 240 ítemes con cinco opciones de respuesta: siempre, casi siempre, pocas veces, casi nunca y nunca. Cuenta con cuatro escalas que funcionan como indicadores de los trastornos de Anorexia Nervosa, Bulimia, conductas de atracones de comida (binge eating) y conductas de oscilación con dietas (yo-yo dieting). Posee además 13 escalas de factores asociados: 7 del área somática y 5 del área psíquica y familiar. Las escalas de factores asociados, sus aspectos interpretativos y cantidad de ítemes se presentan en el Cuadro 3.

La autora reporta niveles de confiabilidad de la prueba dados por coeficientes de consistencia interna Alfa de Cronbach. La prueba total muestra un nivel de confiabilidad $a=.85$, las escalas presentan niveles de confiabilidad entre .70 y .92 . En cuanto a la validez del instrumento, la autora señala que el ICA presenta validez de constructo dado por el método de análisis factorial. Las trece escalas constituyen dos 
Estudio epidemiológico de los trastornos alimentarios y factores asociados...

Cuadro 3

Escalas de factores asociados del ICA

\begin{tabular}{|c|c|c|}
\hline Escala & Aspectos evaluados & Itemes \\
\hline \multicolumn{2}{|c|}{ Area somática } & 155 \\
\hline Dietas & $\begin{array}{l}\text { Organizadores de las actividades diarias, creencias asociadas, } \\
\text { posibilidades idealizadas del control del hambre, consecuencias } \\
\text { de mantener o romper una dieta. }\end{array}$ & 37 \\
\hline Comida & $\begin{array}{l}\text { Funciones, relaciones con las emociones, distorsiones perceptuales, } \\
\text { conductas patológicas, mecanismos de control y evitación. }\end{array}$ & 48 \\
\hline Peso & $\begin{array}{l}\text { Percepción y preocupación por el mismo, rutinas, temores, } \\
\text { controles, conductas patológicas asociadas. }\end{array}$ & 20 \\
\hline $\begin{array}{l}\text { Laxantes, } \\
\text { diuréticos y } \\
\text { medicinas }\end{array}$ & Uso y abuso de medicamentos purgativos y conductas asociadas. & 13 \\
\hline \begin{tabular}{l|} 
Vómitos \\
y periodos \\
menstruales
\end{tabular} & $\begin{array}{l}\text { Presencia o ausencia de dichas conductas, frecuencia y creencias } \\
\text { asociadas, relación entre el peso y los periodos menstruales, } \\
\text { amenorrea y conductas compartidas con las compañeras. }\end{array}$ & 11 \\
\hline $\begin{array}{l}\text { Cuerpo y } \\
\text { ejercicio }\end{array}$ & $\begin{array}{l}\text { Creencias asociadas al cuerpo, a la delgadez y a la imagen } \\
\text { corporal, autoestima y la función purgativa de los ejercicios. }\end{array}$ & 16 \\
\hline Salud & Estado de salud en general. & 10 \\
\hline \multicolumn{2}{|c|}{ Area psíquica y familiar } & 115 \\
\hline Sexualidad & $\begin{array}{l}\text { Actitudes, creencias e intereses, manejo y relación con los } \\
\text { métodos anticonceptivos. }\end{array}$ & 8 \\
\hline Sentimientos & $\begin{array}{l}\text { La relación entre el descontrol, la tristeza, la incertidumbre, } \\
\text { el miedo, la soledad, el vacío emocional y la comida; el uso } \\
\text { de la comida como respuesta de gratificación y satisfacción } \\
\text { instantánea de los afectos. }\end{array}$ & 21 \\
\hline Personalidad & $\begin{array}{l}\text { Análisis de la estructuración y coherencia del self, así como } \\
\text { su manejo ante situaciones de éxito, aceptación de las normas, } \\
\text { frustración y descontrol. }\end{array}$ & 54 \\
\hline $\begin{array}{l}\text { Opinión de } \\
\text { los demás }\end{array}$ & $\begin{array}{l}\text { Evaluación de la importancia de los criterios del mundo } \\
\text { externo, del grupo de pares y de la familia en la consolidación } \\
\text { del self. }\end{array}$ & 7 \\
\hline Familia & $\begin{array}{l}\text { Evaluación de las interacciones, los roles, las jerarquías y las } \\
\text { características del sistema familiar; capacidad para enfrentar } \\
\text { situaciones de conflicto familiar. }\end{array}$ & 25 \\
\hline
\end{tabular}


factores: el de elementos referidos al área somática (dietas, comida, peso, laxantes, vómitos, cuerpo) y el del área psíquica (sexualidad, sentimientos, personalidad, opinión de los demás y familia). La escala de salud aparece asociada a ambos factores. Estos factores explican el 63\% de la varianza total del instrumento (Zusman, 2000).

A partir de la aplicación del instrumento y de un análisis fino del mismo, surgió la necesidad de incorporar una escala adicional, a la que se ha denominado Normas y que contiene los ítemes más puntuados por las jóvenes de la muestra, reflejando así un perfil de lo que sienten y piensan con respecto a su cuerpo, su peso, la comida, sus afectos y personalidad. Esta escala adicional quedó finalmente compuesta por 12 ítemes. Entre los cuales se encuentran:

- siento que la comida controla mi vida,

- trato de comer comida light,

- me gusta que mi estómago esté vacío,

- pienso mucho antes de comer alimentos que engordan,

- me disgusta la imagen que tengo de mi cuerpo,

- las mujeres queremos estar más flacas.

Por otra parte, la aplicación del ICA mostró algunas dificultades estadísticas en cuanto a la utilización de la escala de Sexualidad, debido a lo cual se optó por eliminar dicha escala de la interpretación de los factores asociados.

Para la definición de los criterios diagnósticos se identificó un conjunto de ítemes críticos. Estos son:

- (3) Tomo diuréticos

- (22) Tomo laxantes

- (32) Fracaso en mis intentos repetidos de hacer dietas

- (62) Vomito después de comer

- (67) Tomo diuréticos cuando siento mi cuerpo hinchado 
Estudio epidemiológico de los trastornos alimentarios y factores asociados...

- (85) Tengo atracones de comida

- (105) Vomito a pesar de estar a dieta

- (107) Se me suspende la regla cuando bajo de peso

- (113) Evito la comida

- (119) Organizo mi vida en función a las dietas

- (139) Utilizo laxantes para controlar mi peso

- (179) Hago ejercicios físicos para mantenerme delgada

- (198) Hago ejercicios para quemar calorías

- (208) Evito comer a pesar de tener hambre

A partir de ellos y de acuerdo a los criterios considerados en el DSMIV (APA, 1994), se estableció un conjunto de criterios diagnósticos para cada uno de los trastornos anteriormente descritos. En el Cuadro 4 se presentan estos criterios.

\section{Cuadro 4}

Criterios diagnósticos del ICA

\begin{tabular}{|l|l|}
\hline Trastornos & \multicolumn{1}{|c|}{ Criterios diagnósticos del ICA } \\
\hline Anorexia & Item $107>3$ y (Item $113>3$ y/o Item $208>3)$ \\
Bulimia & Item $85>3$ y (Item $3>3$ y/o Item $22>3$ y/o Item $62>2$ y/o Item \\
& $\begin{array}{l}67>2 \text { y/o Item } 105>2 \text { y/o Item } 139>2 \text { y/o Item } 179>3 \text { y/o Item } \\
198>3)\end{array}$ \\
Binge eating & $\begin{array}{l}\text { Item } 85>3 \text { y (Item } 3<4 \text { y Item } 22<4 \text { y Item } 62<3 \text { y Item } 67< \\
3 \text { y Item } 105<3 \text { y Item } 139<3 \text { y Item } 179<4 \text { y Item } 198<4)\end{array}$ \\
Yo-yo dieting & Item 32 $>2$ y Item $119>2$ \\
\hline
\end{tabular}

Para la definición de los criterios de riesgo, en primer lugar se seleccionaron un conjunto de ítemes críticos y un punto de corte de acuerdo a la escala Likert, además de algunas agrupaciones o clusters de indicadores. Luego, de acuerdo a la presencia o ausencia de cada uno de estos indicadores, se realizó una sumatoria que se transformó a puntajes T. Para determinar el riesgo se consideraron los casos que presentaron una $T>60$. De manera adicional se consideró importante incluir un criterio general de riesgo que se incluye en el Cuadro 5. 
Patricia Martínez, Lillyana Zusman, Joan Hartley, Roxanna Morote y Arturo Calderón

\section{Cuadro 5}

Criterios de riesgo del ICA

\begin{tabular}{|c|c|}
\hline Trastornos & Criterios de Riesgo del ICA \\
\hline Anorexia & $\begin{array}{l}\text { ([Item } 9, \text { Item } 23, \text { Item } 25, \text { Item } 31 \text {, Item } 94, \text { Item } 107, \text { Item } 113, \\
\text { Item } 116, \text { Item } 120, \text { Item } 123, \text { Item } 136, \text { Item } 208]>3)+(\text { Item } 209 \\
>2)+([\text { Item } 134>3 \text { o Item } 148>3] \text { y [Item } 33<3 \text { y Item } 203< \\
3])+(\text { Item } 137>3 \text { y Item } 158>3)\end{array}$ \\
\hline Bulimia & $\begin{array}{l}([\text { Item } 3 \text {, Item } 22 \text {, Item } 43 \text {, Item } 171 \text {, Item } 179, \text { Item } 183 \text {, Item } 198, \\
\text { Item } 211 \text {, Item } 230, \text { Item } 234]>3)+([\text { Item } 57, \text { Item } 62, \text { Item } 67, \\
\text { Item } 87 \text {, Item } 90 \text {, Item } 105 \text {, Item } 112 \text {, Item } 139 \text {, Item } 145]>2)+ \\
\text { (Item } 85>3 \text { y [Item } 3>3 \text { o Item } 22>3 \text { o Item } 62>2 \text { o Item } 67> \\
2 \text { o Item } 105>2 \text { o Item } 139>2 \text { o Item } 179>3 \text { o Item } 198>3])+ \\
([\text { Item } 134>3 \text { o Item } 148>3] \text { y [Item } 33>3 \text { o Item } 203>3])\end{array}$ \\
\hline Binge eating & $\begin{array}{l}\text { Item } 41>3+\text { Item } 93>3+(\text { Item } 85>3 \text { y }[\text { Item } 3<4 \text { y Item } 22< \\
4 \text { y Item } 62<3 \text { y Item } 67<3 \text { y Item } 105<3 \text { y Item } 139<3 \text { y Item } \\
179<4 \text { y Item } 198<4 \text { ]) }\end{array}$ \\
\hline Yo-yo dieting & $\begin{array}{l}([\text { Item } 17, \text { Item } 28, \text { Item } 37, \text { Item } 46, \text { Item } 49, \text { Item } 59, \text { Item } 82, \\
\text { Item } 101, \text { Item } 149, \text { Item } 164]>3)+([\text { Item } 32, \text { Item } 119]>2)\end{array}$ \\
\hline General & (Todos los anteriores) + Item $4>3$ \\
\hline
\end{tabular}

\section{Procedimiento}

Una vez seleccionados los centros educativos, se procedió a seleccionar a las participantes de la investigación de acuerdo a los criterios establecidos. Las aplicaciones del ICA las realizaron cuatro psicólogas entrenadas, quienes administraron la prueba a todas las adolescentes que aceptaron participar en la investigación. Al concluirse la administración del inventario se invitó a las participantes a ser pesadas y medidas. Sólo el $85,3 \%$ de la muestra accedió a ser medida y el $84,1 \%$ accedió a ser pesada.

Luego se elaboró la base de datos depurando los inventarios que no fueron contestados de forma correcta y se procedió a realizar los análisis estadísticos. Para ello se elaboraron índices diagnósticos para los cuatro cuadros que mide la prueba (anorexia, bulimia, binge eating y yo-yo dieting) y un índice global de diagnóstico, en base a 
criterios teóricos y a la presencia de ítemes críticos. En base a los datos de peso y estatura se elaboró el Indice de Masa Corporal para incluirlo como criterio de análisis.

\section{Resultados}

\section{Indice de Masa Corporal}

En términos generales se encontró que existe una diferencia significativa entre el peso y la talla real y entre el peso y la talla ideal. Mientras que para el peso la diferencia es de $5.29 \%$, la diferencia de talla es del $10.5 \%$. Estos datos sugieren que las jóvenes evaluadas muestran cierto descontento, tanto con su peso como con su talla. Esta diferencia entre lo real y lo ideal tiene también una incidencia sobre el Indice de Masa Corporal (IMC) de tal manera que el valor real es de 20.87, dato que coincide con los valores adecuados propuestos por la Organización Mundial de la Salud (OMS), y el ideal es de 17.71, valor que está por debajo de lo normativo y saludable.

En el Cuadro 6 se presenta el IMC real y deseado de las participantes que accedieron a ser medidas y pesadas de acuerdo a las categorías descritas por la OMS.

\section{Cuadro 6}

IMC real y deseado

\begin{tabular}{|l|cc|cc|cc|cc|cc|}
\hline \multirow{3}{*}{ IMC Real } & \multicolumn{10}{|c|}{ IMC Deseado } \\
\cline { 2 - 12 } & \multicolumn{1}{|c|}{ B. P. severo } & \multicolumn{2}{|c|}{ Bajo peso } & \multicolumn{2}{|c|}{ Peso normal } & \multicolumn{2}{|c|}{ Sobrepeso } & \multicolumn{2}{|c|}{ Total } \\
\cline { 2 - 12 } & $f$ & $\%$ & $f$ & $\%$ & $f$ & $\%$ & $f$ & $\%$ & $f$ & $\%$ \\
\hline Bajo peso severo & 109 & 66.1 & 39 & 23.6 & 17 & 10.3 & & & 165 & 100 \\
Bajo peso & 226 & 59.6 & 107 & 28.2 & 46 & 12.1 & & & 379 & 100 \\
Peso normal & 455 & 56 & 247 & 30.4 & 108 & 13.3 & 3 & 0.4 & 813 & 100 \\
Sobrepeso & 53 & 43.1 & 35 & 28.5 & 34 & 27.6 & 1 & 0.8 & 123 & 100 \\
Obesidad moder. & 1 & 12.5 & 4 & 50 & 2 & 25 & 1 & 12.5 & 8 & 100 \\
\hline \multicolumn{1}{|c|}{ Total } & 844 & 56.7 & 432 & 29 & 207 & 13.9 & 5 & 0.3 & 1488 & 100 \\
\hline
\end{tabular}


Patricia Martínez, Lillyana Zusman, Joan Hartley, Roxanna Morote y Arturo Calderón

Aquí se puede observar en primer lugar que el $85.7 \%$ de las participantes desea tener un peso inferior al normal para su talla, de las cuales el $56.7 \%$ desea tener un bajo peso severo. Por otro lado se puede ver que sólo un $13.3 \%$ de las que presentan un nivel de peso normal se encuentra satisfecha con su peso.

Un dato adicional lo constituyen las diferencias encontradas en los niveles de peso y talla ideales de las alumnas de colegios públicos y privados. Así, se encontró que mientras en las adolescentes de colegios privados predomina el descontento con el peso, en las de colegios públicos predomina el descontento con la talla.

\section{Prevalencia}

La prevalencia de los trastornos alimentarios, de acuerdo a los criterios diagnósticos del ICA, se presenta en el Cuadro 7. Aquí se están considerando tanto los trastornos que se presentan de forma independiente, como los que se presentan combinados. En total se encontraron 351 casos $(16.4 \%)$ con uno o más trastornos alimentarios, de los cuales 62 casos (2.9\%) presentaron más de un diagnóstico.

Estos datos ponen en evidencia la alta prevalencia del yo-yo dieting en la población adolescente así como la presencia significativa de la bulimia y de las conductas binge eating. La anorexia nervosa, por el contrario, aparece sólo en 31 casos, de los cuales sólo 9 presentan una anorexia pura.

Asimismo, se puede observar que las jóvenes evaluadas tienden a presentar cuadros mixtos en los que se intercala el descontrol de comer desaforadamente y el control de restringir el exceso, sea a través de las purgas o de las dietas estrictas que, por lo general, como ellas mismas informan, no llegan a completar su fin y, por lo tanto, instalan circuitos repetidos que oscilan entre el comer y dejar de comer, circuitos que pueden terminar por dominar la vida de estas jóvenes, dato que se 
Estudio epidemiológico de los trastornos alimentarios y factores asociados...

\section{Cuadro 7}

Prevalencia de trastornos alimentarios

\begin{tabular}{|ll|cc|}
\hline Trastorno & $f$ & $\%$ \\
\hline Anorexia & Anorexia & 9 & $0.4 \%$ \\
& Anorexia/Bulimia & 2 & $0.1 \%$ \\
& Anorexia/Yo-yo dieting & 10 & $0.5 \%$ \\
& Anorexia/Bulimia/Yo-yo dieting & 10 & $0.5 \%$ \\
& Total & $\mathbf{3 1}$ & $\mathbf{1 . 4 \%}$ \\
\hline Bulimia & Bulimia & 64 & $3 \%$ \\
& Bulimia/Yo-yo dieting & 35 & $1.6 \%$ \\
& Bulimia/Anorexia & 2 & $0.1 \%$ \\
& Bulimia/Anorexia/Yo-yo dieting & 10 & $0.5 \%$ \\
& Total & $\mathbf{1 1 1}$ & $\mathbf{5 . 2 \%}$ \\
\hline Binge eating & Binge eating & 79 & $3.7 \%$ \\
& Binge eating/Yo-yo dieting & 5 & $0.2 \%$ \\
& Total & $\mathbf{8 4}$ & $\mathbf{3 . 9 \%}$ \\
\hline Yo-yo dieting & Yo-yo dieting & 137 & $6.4 \%$ \\
& Yo-yo dieting/Anorexia & 10 & $0.5 \%$ \\
& Yo-yo dieting/Bulimia & 35 & $1.6 \%$ \\
& Yo-yo dieting/Binge eating & 5 & $0.2 \%$ \\
& Yo-yo dieting/Anorexia/Bulimia & 10 & $0.5 \%$ \\
& Total & $\mathbf{1 9 7}$ & $\mathbf{9 . 2 \%}$ \\
\hline
\end{tabular}

corrobora a través del análisis de frecuencia del item "siento que mi vida se organiza en función de las dietas".

\section{Población en riesgo}

A continuación se presentan los resultados de la población identificada como población en riesgo de presentar trastornos alimentarios, en base a los criterios anteriormente descritos. El nivel de riesgo en general, sin especificación de cuadros corresponde al $15.1 \%(n=323)$ del grupo total. 
Patricia Martínez, Lillyana Zusman, Joan Hartley, Roxanna Morote y Arturo Calderón

\section{Cuadro 8}

Niveles de riesgo en la muestra

\begin{tabular}{|lcc|}
\hline Trastorno & $f$ & $\%$ \\
\hline Anorexia & 343 & $16 \%$ \\
Bulimia & 256 & $12 \%$ \\
Binge eating & 394 & $18.4 \%$ \\
Yo-yo dieting & 371 & $17.3 \%$ \\
\hline
\end{tabular}

Los niveles de riesgo encontrados en las escolares muestran que los trastornos clásicos (anorexia y bulimia) tienen niveles inferiores a los que corresponden a los llamados trastornos no específicos (binge eating y yo-yo dieting) (Cuadro 8). Las proporciones en cada cuadro y de riesgo en general son muy superiores a los cuadros diagnósticos, lo que da cuenta de una presencia importante de comportamientos y sentimientos distorsionados en una cantidad significativa de jóvenes. Estas jóvenes se encuentran atravesando condiciones comportamentales y emocionales que ponen en riesgo su bienestar actual y su desarrollo posterior, pudiendo llegar a presentar síntomas propios de los cuadros clínicos.

\section{Tipo de colegio}

Se analizó la presencia de cuadros diagnósticos en los grupos en función al tipo de colegio al que pertenecen las participantes (de gestión privada o pública). Se encontró que el 15,4\% del grupo de participantes pertenecientes a colegios públicos presenta uno o más trastornos alimentarios, mientras que en los colegios privados este porcentaje alcanza un $18,9 \%$. De este total, el $3.1 \%$ de colegios públicos y el $4.3 \%$ de colegios privados presenta más de un trastorno. En el Cuadro 9 se presenta la frecuencia y porcentaje de participantes con uno o más trastornos alimentarios según su tipo de colegio. 
Estudio epidemiológico de los trastornos alimentarios y factores asociados...

\section{Cuadro 9}

Prevalencia de trastornos alimentarios de acuerdo al tipo de colegio

\begin{tabular}{|ll|cccc|}
\hline \multirow{2}{*}{ Trastorno } & \multicolumn{3}{|c|}{$\begin{array}{c}\text { Público } \\
(\mathrm{n}=1555)\end{array}$} & $\begin{array}{c}\text { Privado } \\
(\mathrm{n}=586)\end{array}$ \\
\cline { 3 - 6 } & & $f$ & $\%$ & $f$ & $\%$ \\
\hline Anorexia & Anorexia & 8 & $0.5 \%$ & 1 & $0.2 \%$ \\
& Anorexia/Bulimia & 1 & $0.1 \%$ & 1 & $0.2 \%$ \\
& Anorexia/Yo-yo dieting & 6 & $0.4 \%$ & 4 & $0.7 \%$ \\
& Anorexia/Bulimia/Yo-yo dieting & 8 & $0.5 \%$ & 2 & $0.3 \%$ \\
& Total & $\mathbf{2 3}$ & $\mathbf{1 . 5 \%}$ & $\mathbf{8}$ & $\mathbf{1 . 4 \%}$ \\
\hline Bulimia & Bulimia & 45 & $2.9 \%$ & 19 & $3.2 \%$ \\
& Bulimia/Yo-yo dieting & 21 & $1.4 \%$ & 14 & $2.4 \%$ \\
& Bulimia/Anorexia & 1 & $0.1 \%$ & 1 & $0.2 \%$ \\
& Bulimia/Anorexia/Yo-yo dieting & 8 & $\mathbf{0 . 5 \%}$ & 2 & $0.3 \%$ \\
& Total & $\mathbf{7 5}$ & $\mathbf{4 . 8 \%}$ & $\mathbf{3 6}$ & $\mathbf{6 . 1 \%}$ \\
\hline Binge eating & Binge eating & 46 & $3 \%$ & 33 & $5.6 \%$ \\
& Binge eating/Yo-yo dieting & 3 & $\mathbf{0 . 2 \%}$ & 2 & $0.3 \%$ \\
& Total & $\mathbf{4 9}$ & $\mathbf{3 . 2 \%}$ & $\mathbf{3 5}$ & $\mathbf{6 . 0 \%}$ \\
\hline Yo-yo dieting & Yo-yo dieting & $\mathbf{1 0 2}$ & $\mathbf{6 . 6 \%}$ & 35 & $6.0 \%$ \\
& Yo-yo dieting/Anorexia & 6 & $0.4 \%$ & 4 & $0.7 \%$ \\
& Yo-yo dieting/Bulimia & 21 & $1.4 \%$ & 14 & $2.4 \%$ \\
& Yo-yo dieting/Binge eating & 3 & $\mathbf{0 . 2 \%}$ & 2 & $0.3 \%$ \\
& Yo-yo dieting/Anorexia/Bulimia & 8 & $\mathbf{0 . 5 \%}$ & 2 & $0.3 \%$ \\
& Total & $\mathbf{1 4 0}$ & $\mathbf{9 \%}$ & $\mathbf{5 7}$ & $\mathbf{9 . 7 \%}$ \\
\hline
\end{tabular}

Aquí puede observarse en primer lugar una alta incidencia de trastornos alimentarios en el grupo de colegios públicos. Esto confirma la idea del incremento de estos trastornos también en niveles socioeconómicos bajos, aunque se mantiene una proporción mayor en las alumnas de colegios privados, especialmente en binge eating y en bulimia.

El nivel de riesgo también fue explorado de acuerdo al tipo de colegio, estos resultados se presentan en la Figura 2. En términos generales, las alumnas de colegios privados presentan mayor proporción de conductas de riesgo de trastornos alimentarios en general (16\%, 
$\mathrm{n}=94)$ que las adolescentes de colegios públicos $(14.7 \%, \mathrm{n}=229)$. Sin embargo, los niveles de riesgo a presentar trastornos de anorexia y bulimia en las escolares de colegios públicos son mayores. Las adolescentes de colegios privados presentan un mayor riesgo en cuanto a los trastornos no específicos, binge eating y yo-yo dieting.

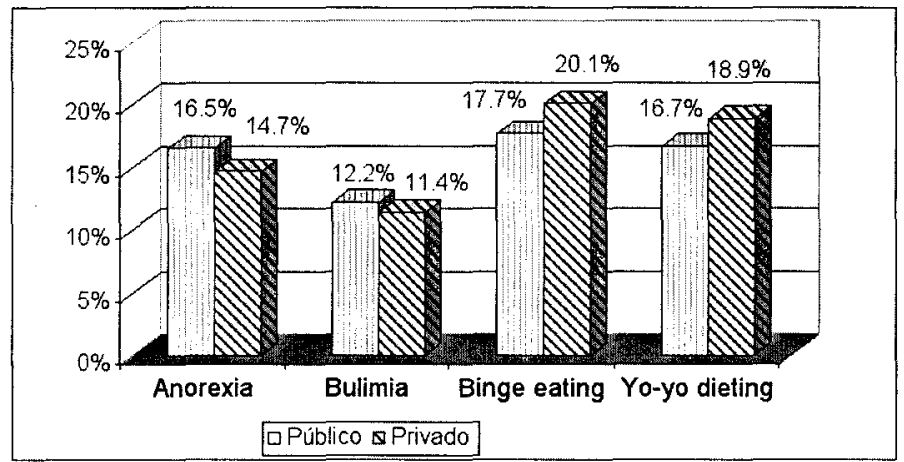

Figura 2. Niveles de riesgo de acuerdo al tipo de colegio

Vemos que en el caso de la anorexia, la tendencia es similar a la encontrada en los casos con diagnóstico clínico. Es decir, se encuentra un mayor número de jóvenes con este trastorno o en riesgo en los colegios públicos de Lima. En general, la variedad de la distribución del nivel de riesgo por cuadro en el caso de los colegios públicos y privados, da cuenta de un fenómeno de homogenización de las conductas distorsionadas de la alimentación en las adolescentes.

\section{Factores asociados}

Con el fin de explorar los factores de personalidad asociados a los trastornos alimentarios se analizaron las escalas del ICA en aquellos casos que presentaron algún diagnóstico de trastorno alimentario y se compararon con los que no presentaron ningún diagnóstico mediante la $U$ de Mann Whitney. Se encontró que los grupos diagnosticados con algún trastorno presentaron diferencias significativas frente a los grupos 
Estudio epidemiológico de los trastornos alimentarios y factores asociados...

que no presentaron ningún diagnóstico, en todas las escalas. En base a este resultado y para identificar con mayor precisión el nivel de diferencia entre las medias se procedió a utilizar el estadístico $d$ de Cohen, con el fin de determinar el tamaño de las diferencias.

En el Cuadro 10 se presenta la comparación de medias de las escalas del ICA y su nivel de diferencia en función a la presencia de algún diagnóstico encontrado.

Cuadro 10

Escalas del ICA de acuerdo a la presencia o ausencia de trastornos alimentarios

\begin{tabular}{|l|cccc|cc|}
\hline \multirow{1}{*}{ Escala } & \multicolumn{2}{|c|}{$\begin{array}{c}\text { Sin trastorno } \\
(\mathrm{n}=1790)\end{array}$} & \multicolumn{2}{c|}{$\begin{array}{c}\text { Con trastorno } \\
(\mathrm{n}=351)\end{array}$} & & \\
\cline { 2 - 7 } & Media & $D E$ & Media & $D E$ & $U$ & $d$ \\
\hline Dieta & 41.2 & 13.9 & 63.7 & 18.1 & 108518 & $1.40^{* * *}$ \\
Comida & 81.0 & 20.8 & 117.3 & 24.5 & 85593.5 & $1.60^{* * *}$ \\
Peso & 35.5 & 8.8 & 46.7 & 9.4 & 121315 & $1.23^{* * *}$ \\
Laxantes & 19.7 & 4.5 & 25.3 & 10.0 & 197454.5 & $0.78^{* *}$ \\
Vómitos & 14.9 & 4.5 & 19.8 & 7.8 & 186712 & $0.80^{* *}$ \\
Cuerpo & 46.4 & 13.0 & 64.1 & 14.5 & 115513.5 & $1.29^{* * *}$ \\
Salud & 16.0 & 4.6 & 20.6 & 6.2 & 174493.5 & $0.85^{* * *}$ \\
Sentimientos & 46.1 & 10.9 & 55.4 & 9.9 & 167586 & $0.89^{* * *}$ \\
Personalidad & 135.4 & 22.4 & 154.8 & 20.2 & 165787.5 & $0.91^{* * *}$ \\
Opinión & 21.5 & 4.7 & 25.3 & 4.6 & 177390 & $0.80^{* *}$ \\
Familia & 66.7 & 16.8 & 77.4 & 16.1 & 201250 & $0.65^{* *}$ \\
Normas & 37.5 & 11.1 & 52.3 & 12.1 & 117443.5 & $1.27^{* * *}$ \\
\hline
\end{tabular}

*** diferencia grande, $* *$ diferencia mediana, $*$ diferencia pequeña

Aquí se puede observar que las escalas que corresponden al área somática son las que se ven más afectadas (o muestran mayor nivel de perturbación frente a los grupos asintomáticos). Es decir, las escalas de Dietas, Comida, Peso, Cuerpo y Salud señalan puntajes mucho más elevados en las jóvenes con diagnóstico que en las que no lo tienen. Las conductas que reflejan estas escalas dan cuenta de conductas muy distorsionadas en cuanto al cuidado del cuerpo. Asimismo, del área 
psíquica y familiar destacan las escalas de Sentimientos, Personalidad y Normas, asociadas a sentimientos de descontrol, frustración y falta de coherencia en el self.

En el Cuadro 11 se presenta el nivel de diferencias en las escalas del ICA de acuerdo a la presencia o ausencia de trastornos específicos (anorexia, bulimia, binge eating y yo-yo dieting).

\section{Cuadro 11}

Nivel de diferencias en las escalas del ICA de acuerdo a diagnósticos especificos

\begin{tabular}{|l|cccc|}
\hline Escala & Anorexia & Bulimia & Binge eating & Yo-yo dieting \\
\hline Dieta & $1.97^{* * *}$ & $1.27^{* * *}$ & 0.03 & $2.52^{* * *}$ \\
Comida & $2.13^{* * *}$ & $1.75^{* * *}$ & $0.49^{*}$ & $2.02^{* * *}$ \\
Peso & $1.68^{* * *}$ & $1.23^{* * *}$ & $0.23^{*}$ & $1.86^{* * *}$ \\
Laxantes & $1.26^{* * *}$ & $1.13^{* * *}$ & 0.13 & $0.96^{* * *}$ \\
Vómitos & $1.49^{* * *}$ & $1.09^{* * *}$ & 0.13 & $1.03^{* * *}$ \\
Cuerpo & $1.68^{* * *}$ & $1.34^{* * *}$ & $0.40^{*}$ & $1.71^{* * *}$ \\
Salud & $1.80^{* * *}$ & $1.06^{* * *}$ & $0.48^{*}$ & $0.82^{* * *}$ \\
Sentimientos & $1.29 * * *$ & $0.94^{* * *}$ & $0.64^{* *}$ & $0.88^{* * *}$ \\
Personalidad & $1.17^{* * *}$ & $0.91^{* * *}$ & $0.59^{* *}$ & $0.94^{* * *}$ \\
Opinión & $0.96^{* * *}$ & $0.85^{* * *}$ & $0.37^{*}$ & $0.96^{* * *}$ \\
Familia & $0.84^{* * *}$ & $0.63^{* *}$ & $0.30^{*}$ & $0.75^{* *}$ \\
Normas & $1.83^{* * *}$ & $1.18^{* * *}$ & 0.17 & $2.07^{* * *}$ \\
\hline
\end{tabular}

$* * * \mathrm{~d}$ grande, $* *$ d mediana, $* \mathrm{~d}$ pequeña

Resulta clara aquí la elevación significativa de todas las escalas en los cuadros de anorexia, bulimia y yo-yo dieting. Los casos de binge eating sólo presentan diferencias medianas en Sentimientos y Personalidad.

Asimismo, se presenta la comparación de medias de las escalas del ICA y su nivel de diferencia en función a la presencia o ausencia de indicadores de riesgo en general (Cuadro 12). 
Estudio epidemiológico de los trastornos alimentarios y factores asociados...

\section{Cuadro 12}

Escalas del ICA de acuerdo a presencia o ausencia de riesgo

\begin{tabular}{|l|cccccc|}
\hline \multirow{2}{*}{ Escala } & \multicolumn{6}{|c}{$\begin{array}{c}\text { Sin Riesgo } \\
(\mathrm{n}=1818)\end{array}$} \\
\cline { 2 - 7 } & Media & $D E$ & Media & DE & $U$ & $d$ \\
\hline Dieta & 40.2 & 12.9 & 71.2 & 11.6 & 27590 & $2.52^{* * *}$ \\
Comida & 80.2 & 19.6 & 125.3 & 18.9 & 31966.5 & $2.35 * * *$ \\
Peso & 35.0 & 8.4 & 50.7 & 6.1 & 41748 & $2.18^{* * *}$ \\
Laxantes & 19.3 & 3.9 & 27.9 & 10.3 & 122081.5 & $1.22^{* * *}$ \\
Vómitos & 14.5 & 3.9 & 22.5 & 7.7 & 101239 & $1.37^{* * *}$ \\
Cuerpo & 45.7 & 12.3 & 69.4 & 10.8 & 47407.5 & $2.05^{* * *}$ \\
Salud & 15.9 & 4.6 & 21.3 & 5.9 & 137392.5 & $1.03^{* * *}$ \\
Sentimientos & 46.1 & 10.8 & 56.2 & 10.3 & 147681.5 & $0.95^{* * *}$ \\
Personalidad & 135.4 & 22.1 & 156.7 & 20.8 & 143284 & $1.00^{* * *}$ \\
Opinión & 21.5 & 4.6 & 26.0 & 4.2 & 139267.5 & $1.02^{* * *}$ \\
Familia & 66.5 & 16.4 & 79.6 & 17.0 & 169383.5 & $0.78^{* *}$ \\
Normas & 36.8 & 10.5 & 57.3 & 8.1 & 40661 & $2.20^{* * *}$ \\
\hline
\end{tabular}

*** diferencia grande, ** diferencia media, * diferencia pequeña

En este cuadro se puede ver que en todas las escalas las diferencias son importantes y que por lo tanto se puede asumir que se encuentran asociadas a los niveles de riesgo.

\section{Discusión}

En los últimos 30 años se ha podido apreciar en la sociedad occidental la alta incidencia de desórdenes alimenticios. La historia del desarrollo de la psicopatología pone en evidencia que la prevalencia de las enfermedades mentales ocurre en un tiempo histórico social. En este sentido, se puede sugerir que la sociedad contemporánea deposita en la comida un flujo desplazado de funciones y utiliza el cuerpo (femenino) como depositario de un conjunto de ansiedades que de otra manera quedarian, probablemente, libres de todo sostén. 
La representación mental del cuerpo es un proceso largo que no tiene un tiempo fijo; se sabe cuando comienza pero no cuando termina. En el pasaje de la pubertad a la adolescencia el cuerpo sufre una serie de modificaciones sustanciales que tienen una representación en la formación de la identidad y de la identidad de género. En los últimos años, este proceso natural está siendo interferido por la presencia de imágenes femeninas idealizadas que se han constituido en un estereotipo del deber ser mujer a través de las exigencias de una sociedad que define al cuerpo en función de ideales socio culturales que validan la apariencia como el modo de ser.

El peso que, como número objetivo y medible, debería ser un determinante objetivo del cuerpo, ha devenido en un dato irrelevante ya que los criterios de delgadez y de gordura reciben, actualmente, interpretaciones subjetivas determinadas por la cultura. Este proceso acarrea, lamentablemente, consecuencias emocionales que derivan en que la formación de la identidad y de la identidad de género se vea afectada por la vivencia de estar en un cuerpo que oscila entre sus posibilidades y dificultades de acercarse y/o alejarse al ideal establecido por la cultura. Ante la dificultad de establecer una combinación adecuada de lo somático y lo psíquico, aparece como respuesta natural, ampliamente validada por la sociedad contemporánea, el reforzamiento de la apariencia que, a menos que sea temporal y que se organice paulatinamente en la forma de una imagen corporal adecuada, podría verse atrapada en el desencuentro permanente de los aspectos físicos y somáticos que deriva en una fragmentación del self.

Es conocido que las personas que padecen un trastorno de alimentación no sólo establecen un patrón alimentario alejado de los procesos fisiológicos normales de hambre y saciedad, sino que también adoptan una actitud enajenante hacia su propio cuerpo, estableciéndose éste como un elemento angustiante que genera insatisfacción y disgusto (Rausch, 1996). 
Estudio epidemiológico de los trastornos alimentarios y factores asociados...

En este sentido, resulta interesante subrayar que se encuentra una diferencia en el sentir de las participantes con respecto a su talla y peso. $\mathrm{Si}$ bien aparece un descontento general en la mayor parte de la población, resulta significativo que las alumnas de colegios privados están más preocupadas por su peso y que las jóvenes de colegios nacionales están preocupadas y disconformes con su talla y, en consecuencia, en las posibles repercusiones profesionales de su estatura en tanto esta realidad podría frustrar sus deseos e ilusiones de ingresar a los grupos femeninos de las instituciones armadas o a los equipos nacionales de fútbol y básquet, todas ocupaciones que son sentidas como prestigiosas según sus propios testimonios. Pensamos que es importante recordar que la población peruana, mestiza en su mayoría, tiene, por naturaleza, baja talla. Nos preguntamos qué implicancias psicológicas puede conllevar la frustración de no tener una talla alta cuando los requisitos exigidos por ciertos trabajos demandan un perfil que no corresponde al de la población. La coincidencia de estos resultados con los obtenidos en una investigación anterior (Zusman, 2000) pone de manifiesto que se trata de un problema que está inscrito en la población adolescente de Lima Metropolitana.

Frente al cuerpo púber pesado, grande y con hambre se levanta una barrera que define a la comida como dañina y que, simultáneamente, presenta la disposición a "no comer" como logro y como éxito, actitudes que están naturalmente respaldadas por las instituciones sociales. En este sentido, el concepto de dieta se asocia al de comida como un par indivisible, es decir, como las dos caras de una misma moneda. Es más, la gran variedad de publicidad a favor de los cuerpos delgados propone y vende mecanismos alternativos ideales para adelgazar, como son los ejercicios físicos y las purgas en sus formas de vómitos autoinducidos e ingesta de laxantes y diuréticos.

Los resultados de la presente investigación ponen en evidencia la presencia masiva de conductas alimenticias perturbadas en las jóvenes evaluadas, como si los hábitos tradicionales de comer de manera 
ordenada, siguiendo las indicaciones somáticas y cognitivas del hambre real, estuvieran errados o, quizá, extraviados, dando lugar a una forma de comer que oscila en el péndulo del control y el descontrol y que tiene como correlato lo que Zusman (2000) ha denominado hambre emocional. Esto quiere decir que, actualmente, muchas jóvenes comen y dejan de comer por razones de orden emocional y por creencias de orden cognitivo que nada tienen que ver con el correlato somático. Esto se confirma con la alta puntuación en reactivos como los siguientes:

- $\quad$ siento que la comida controla mi vida

- trato de comer comida light

- como cuando estoy triste

- me deprimo cuando como mucho

- la comida me tranquiliza cuando estoy ansiosa

- mi estado de ánimo varía al ritmo de las dietas

- subo y bajo de peso con facilidad

Esta observación nos sugiere que la conducta alimenticia adolescente está modelada por el encuentro entre aspectos individuales (que por determinadas razones se expresan en el cuerpo) y por un conjunto de presiones socio culturales que son parte del paradigma social de nuestro tiempo.

Investigaciones previas realizadas en el Perú (Hartley, 1999; Zusman, 2000) han puesto en evidencia las limitaciones de los criterios diagnósticos planteados por el DSM-IV (APA, 1994) para la evaluación de los trastornos de la alimentación. Ocurre que los criterios biológicos y cognitivos que se plantean no consideran el impacto de los factores emocionales y socioculturales que participan en la gestación y en el mantenimiento de la enfermedad. Es, en este sentido, que sobre la base de la clínica y de los trabajos anteriores realizados con el ICA, se ha determinado un número de ítemes pertenecientes a las diferentes escalas de la prueba, que hemos definido como críticos, en un intento de especificar aspectos puntuales y específicos de cada uno de los 
Estudio epidemiológico de los trastornos alimentarios y factores asociados...

trastornos alimenticios aquí estudiados. Es más, tomando en consideración la escala de Likert (usada en la calificación de la prueba) se han considerado puntuaciones también especificadas como críticas de tal manera que el ítem asociado a la puntuación tiene la doble función de discriminar diagnóstico (en las puntuaciones más elevadas) $\mathrm{y}$ tendencia o riesgo (en las puntuaciones menores).

El yo-yo dieting es un tipo de trastorno alimenticio no reconocido aún por los criterios diagnósticos tradicionales; sin embargo, su nivel de presencia en la población adolescente refleja que la inestabilidad psíquica y somática es una cualidad adolescente contemporánea. No en vano se ha decidido establecer una escala de medición adicional para la prueba que ha sido denominada Normas ya que los ítemes que la componen han recibido puntuaciones altas y constantes en las diversas aplicaciones de la prueba. La falta de estabilidad, de integración y de representación del self determina que estas jóvenes se apeguen a la normatividad social y a los patrones del deber ser que les garantizan un eje de identificación y de solidez así sea a través de la apariencia. Así, nos encontramos con un grupo numeroso de jóvenes que ante la dificultad de organizar los aspectos pulsionales y yoicos han encontrado en la comida una suerte de tobogán por el cual se deslizan periódicamente intentando darle una forma armónica a un cuerpo que no ha conseguido estabilizarse y organizarse adecuadamente después de la pubertad y resolver dificultades más primarias que a consecuencia de no tener un continente más adecuado utilizan el cuerpo (equivocadamente) como tal.

El cuadro diagnóstico del yo-yo dieting refleja una clara distorsión en los aspectos somáticos y psíquicos de las jóvenes evaluadas. Los valores obtenidos en la escala de comida reflejan una suerte de desbalance emocional en la vida de las adolescentes que pone de manifiesto el mecanismo de escisión psique-soma. De esta "dislocación" se desprenden dos consecuencias naturales: ante el aumento de peso, la alternativa es la dieta para modificar un cuerpo 
que se define de acuerdo a los vaivenes de la trilogía establecida entre la comida, el peso y las dietas. Es interesante resaltar que los aspectos de personalidad y de normas aparecen altamente diferenciados respecto de quienes no presentan este diagnóstico. Esta diferencia de medias sugiere que las jóvenes utilizan la trilogía antes mencionada como un paliativo para organizar una identidad en proceso y para manejar situaciones emocionales de control y descontrol de los diferentes aspectos no integrados del self, en tanto que estas jóvenes parecen necesitar de insumos de aprobación y reforzamiento externo para conseguir un mínimo de seguridad interna.

Un dato importante encontrado en la comparación por tipos de colegio señala que las adolescentes de colegios públicos presentan un nivel superior de diagnóstico "puro" de anorexia frente a las adolescentes de colegios privados. Cuando se observa la presencia de anorexia en combinación con otros cuadros diagnósticos, se encuentra que se presenta el mismo fenómeno en el caso de la combinación anorexia/bulimia/yo-yo dieting. La revisión de la literatura mostró que en la actualidad la presencia de anorexia en jóvenes no se encuentra circunscrita a quienes pertenecen a sectores medios o altos de la población. En nuestro medio este fenómeno se comprueba, ya que los niveles de anorexia son muy similares e incluso superiores. Es posible que en la actualidad las barreras sociales que mantenían alejados patrones de vida, modelos culturales (como los asociados a la delgadez) ya no se encuentren delimitados en los sectores altos de la población. Más aún, cuando se habla de una población adolescente, es necesario recordar el impacto significativo que tienen los medios de comunicación y el internet en casi todos los niveles socioeconómicos (excepto tal vez el de la extrema pobreza). Las jóvenes en nuestro medio, independientemente de su nivel socioeconómico, parecen haber asimilado estos patrones.

En los cuadros asociados a conductas más impulsivas y a la presencia de ciclos de conductas extremas en la alimentación (bulimia, binge eating y yo-yo dieting) sí se encuentra que las escolares de 
colegios particulares presentan una mayor proporción de diagnósticos. A diferencia de las primeras, estas jóvenes parecen haber adoptado estrategias más diversas y menos restrictivas. Las jóvenes dan cuenta de un nivel importante de descontrol sobre el manejo de su alimentación, lo que puede reflejar un patrón similar en cuanto a su propia autoestima, identidad, sexualidad y relaciones.

En el análisis de las escalas clínicas resulta evidente la gran diferencia entre las medias de las escalas de dieta, comida, peso y cuerpo, así como en las escalas de personalidad y sentimientos respecto a las demás escalas. Estos datos ponen en perspectiva que la estrecha relación con la comida está ligada a temas afectivos y que, probablemente, estas jóvenes están ajustadas a una normatividad basada en la apariencia y en "deber ser" moderno y actual, que las sostiene y a la vez les reporta satisfacciones y éxito personal.

El cuadro diagnóstico de bulimia presenta una evidente distorsión con la comida y el cuerpo. Estas alteraciones se ponen en relación con el uso de medicinas purgativas (diuréticos y laxantes) y con desórdenes en la personalidad que podrían deberse al pasaje a la acción. El uso reiterativo de medicinas y vómitos se pone al servicio de la gran cantidad de comida que se ingiere así como de los intentos (generalmente frustros) de control a través de las dietas. Consecuencias inmediatas de este desorden se aprecian en el cuerpo, a través de las modificaciones y malestares del mismo, y a través de la salud, en términos de las consecuencias nefastas de la enfermedad, especialmente, en relación a un posible desbalance químico.

El comportamiento de los factores asociados en el caso del diagnóstico exclusivo de binge eating difiere del que se encuentra en los restantes cuadros, en el conjunto de las adolescentes en riesgo y con algún trastorno. Si bien se encuentra que casi todas las escalas de factores asociados (a excepción de laxantes y vómitos) tienen una media superior en el caso de las adolescentes con el diagnóstico, la 
diferencia de las medias no es lo suficientemente significativa para señalar un perfil distinto en este grupo. Es posible que, al tratarse de un trastorno no específico, existan aún algunas controversias en cuanto a la caracterización del mismo. En la actualidad, las características de este trastorno se encuentran aún en exploración. En nuestro medio, estos resultados plantean retos importantes en cuanto a la identificación, caracterización y descripción de las jóvenes con este tipo de trastorno.

Un dato muy importante es que en lo que se refiere a las escalas que pertenecen a las escalas del área psíquica y familiar, la diferencia esperada en las escalas (es decir, la presencia de puntajes altos en las jóvenes con diagnóstico o alto riesgo) no se presenta necesariamente. Las escalas de Sentimientos, Personalidad, Opinión de los Demás y Familia dan cuenta de dinámicas intrapsíquicas y familiares que son comparables en jóvenes, independientemente de los trastornos de alimentación.

El objetivo final del presente trabajo fue poner de manifiesto el comportamiento alimentario de las jóvenes adolescentes de Lima Metropolitana con la finalidad de, por un lado, acercarse a un problema de repercusión mundial que casi ha tomado proporciones epidémicas $y$, por el otro, establecer programas de prevención a través de los colegios y de las instituciones que trabajan con jóvenes. Asimismo, se propone la utilizacion del ICA, no sólo por sus alcances diagnósticos, sino por la posibilidad que ofrece de identificar poblaciones en riesgo, facilitándose así una aplicación masiva y el subsiguiente trabajo de prevención.

\section{Referencias}

Abraham, S. \& Llewellyn-Jones, D. (1997). Eating disorders: The facts (4ta. ed.). Nueva York: Oxford University Press. 
Estudio epidemiológico de los trastornos alimentarios y factores asociados...

Agurto, G. (2000, 31 de Agosto). Anorexia: la dieta mortal. Caretas, p. 51.

American Psychiatric Association. (1994). Diagnostic and statistical manual of mental disorders (4a. ed.). Washington DC.

Bloom, C. \& Kogel, L. (1994). Symbolic meanings of food and body.

En Women's Therapy Center Institute (Ed.), Eating problems: A feminist psychoanalytic treatment model (pp. 57-66). Nueva York: Basic Books.

Bromet, E. (1989). Epidemiología. En A. Bellack \& M. Hersen, Métodos de investigación en psicología clínica. Bilbao: Desclee de Brouwer.

Bruch, H. (1973). Eating disorders: Obesity, anorexia nervosa, and the person within. Nueva York: Basic Books.

Bruch, H. (1994). Conversations with anorexics. Northvale, NJ: Jason Aronson.

Brusset, B. (1977). La anorexia. Madrid: Planeta.

Buena Salud. (1996). Anorexia y bulimia. Buenos Aires: El Obelisco. Byrne, K. (1987). Anorexia and bulimia: Diagnosis. En J. Aronson (Ed.), Insights in the dynamic psychotherapy of anorexia and bulimia (p. 8). Northvale, NJ: Jason Aronson.

Coleman, J.C. (1985). Psicología de la adolescencia. Madrid: Morata. Duker, M. \& Slade, R. (1992). Anorexia nerviosa y bulimia: un tratamiento integrado. México: Limusa.

Fairburn, C. \& Walsh, T. (1995). Atypical eating disorders. En K. Brownell \& C. Fairburn (Eds.), Eating disorders and obesity: A comprehensive handbook (pp. 135-140). Nueva York: Guilford Press.

Fuller, N. (1992). La disputa de la femineidad en el psicoanálisis y las ciencias sociales. Lima: CISEPA-PUCP.

Garfinkel, P. (1995). Classification and diagnosis of eating disorders. En K. Brownell \& C. Fairburn (Eds.), Eating disorders and obesity: A comprehensive handbook (pp. 125-134). Nueva York: Guilford Press. 
Patricia Martínez, Lillyana Zusman, Joan Hartley, Roxanna Morote y Arturo Calderón

Gordon, R. (1990). Anorexia and bulimia: Anatomy of a social epidemic. Cambridge: Blackwell.

Gutwill, S. (1994). Women's eating problems: Social context and the internalization of culture. En Women's Therapy Center Institute (Ed.), Eating problems: A feminist psychoanalytic treatment model (pp. 1-27). Nueva York: Basic Books.

Hartley, J. (1999). Trastornos alimentarios en mujeres adolescentes escolares. Tesis de licenciatura no publicada, Pontificia Universidad Católica del Perú, Lima, Perú.

Hernández, R., Fernández, C. \& Baptista, P. (1998). Metodología de la investigación. México: McGraw Hill.

Hoek, H. W. (1995). The distribution of eating disorders. En K. Brownell \& C. Fairburn (Eds.), Eating disorders and obesity: $A$ comprehensive handbook (pp. 207-212). Nueva York: Guilford Press.

Hsu, G. (1983). The aetiology of anorexia nervosa. Psychological Medicine, 13, 231- 237.

Malson, H. (1998). The thin woman: Feminism, post-structuralism and the social psychology of anorexia nervosa. Nueva York: Routledge.

Perpiñá, C. (1995). Trastornos alimentarios. En A. Belloch, B. Sandín \& F. Ramos (Eds.), Manual de psicopatología, Vol. 1. (pp. 531557). Madrid: McGraw Hill.

Polivy, J. \& Herman, P. (1987). Diagnosis and treatment of normal eating. Journal of Consulting and Clinical Psychology, 55 (5), 635-644.

Polivy, J. \& Herman, P. (1993). Etiology of binge eating: Psychological mechanisms. En C. Fairburn \& T. Wilson (Eds.), Binge eating: Nature, assessment and treatment (pp. 173-205). Nueva York: Guilford Press.

Rausch, C. (1996). La esclavitud de las dietas. Buenos Aires: Paidós. Schwabe, A., Lippe, B., Chang, J., Pops M. \& Yager, J. (1981). Anorexia nervosa. Annals of Internal Medicine, 94, 371-381. 
Estudio epidemiológico de los trastornos alimentarios y factores asociados...

Selvini-Palazzoli, M. (1974). Self-starvation. Nueva York: Jason Aronson.

Selvini-Palazzoli, M. (1988). Mother-daughter relationship. En J. Aronson (Ed.), Insights in the dynamic psychotherapy of anorexia and bulimia (p. 164). Northvale, NJ: Jason Aronson.

Steiner-Adair, C. (1986). The body politic: Normal female adolescent development and the development of eating disorders. Journal of the American Academy of Psychoanalysis, 14 (1), 95-114.

Striegel-Moore, R. (1993). Etiology of binge eating: A developmental perspective. En C. Fairburn \& T. Wilson (Eds.), Binge eating: Nature, assessment and treatment (pp. 144-172). Nueva York: Guilford Press.

Toro, J. \& Villardel, E. (1987). Anorexia nerviosa. Barcelona: Martínez Roca.

Vandereycken, W. \& Meermann, R. (1984). Anorexia nervosa: Is prevention possible? International Journal of Psychiatry in Medicine, 14 (3), 191-205.

Wonderlich, S. (1995). Personality and eating disorders. En K. Brownell \& C. Fairburn (Eds.), Eating disorders and obesity: A comprehensive handbook (pp. 171- 176). Nueva York: Guilford Press.

Zusman, L. (2000). Las conductas alimenticias en adolescentes mujeres de Lima Metropolitana: sus manifestaciones y las características personales y familiares asociadas. Tesis de maestría no publicada, Pontificia Universidad Católica del Perú, Lima, Perú. 